\title{
GRÖBNER $\delta$-BASES AND GRÖBNER BASES FOR DIFFERENTIAL OPERATORS
}

\author{
FRANCISCO J. CASTRO-JIMÉNEZ \\ Facultad de Matemáticas, Universidad de Sevilla \\ Aptdo de Correos 1160, E-41080 Sevilla, Spain \\ E-mail: castro@us.es \\ M. ANGELES MORENO-FRÍAS \\ Facultad de Ciencias, Universidad de Cádiz \\ Río San Pedro s/n, 11510 Puerto Real, Cádiz, Spain \\ E-mail: mariangeles.moreno@uca.es
}

\begin{abstract}
This paper deals with the notion of Gröbner $\delta$-base for some rings of linear differential operators by adapting the works of W. Trinks, A. Assi, M. Insa and F. Pauer. We compare this notion with the one of Gröbner base for such rings. As an application we give some results on finiteness and on flatness of finitely generated left modules over these rings.
\end{abstract}

1. Introduction. We will study Gröbner $\delta$-bases for some rings of linear differential operators.

We have adapted to the differential case some notions and some results obtained by W. Trinks in [TRI] and A. Assi (in [ASS-1] and [ASS-2]) for the case of a commutative polynomial ring with coefficients in a commutative unitary ring.

The notion of Gröbner $\delta$-base we introduce here is equivalent to the one of Gröbner base defined by M. Insa and F. Pauer in [IN-PA]. Nevertheless, we reserve the name Gröbner base for the classical notion introduced in [CAS-1] (see also [CAS-2]). Besides the $\mathbf{k}$-algebras appearing in [IN-PA], the cases $\mathcal{H}=\mathbf{k}[[X]]\left[X^{-1}\right]$ and $\mathcal{H}=\mathbf{k}\{X\}\left[X^{-1}\right]$ (when $\mathbf{k}=\mathbf{R}, \mathbf{C}$ ) will be especially interesting in order to extend the results of [ACG-1] and $[$ ACG-2] to the rings of linear differential operators with coefficients in $\mathcal{H}$.

Section 2 is devoted to the definition of the class of rings of linear differential operators we will study and to the theory of Gröbner $\delta$-bases. We have, in these rings, a reduction

2000 Mathematics Subject Classification: Primary 13N10; Secondary 13P10, 16S32, 32C38, $68 \mathrm{~W} 30$.

Research supported by DGESIC BFM2001-3164, A.I.H.F.2000-0044 and FQM-218.

The paper is in final form and no version of it will be published elsewhere. 
algorithm which allows the effective construction of a Gröbner $\delta$-base for a given ideal, defined by a finite system of generators. This is the aim of sections 3,4 and 5 .

In section 6 we compare the notions of Gröbner $\delta$-base and Gröbner base in the case of the Weyl algebras. We prove that any Gröbner base (in the sense of [CAS-1] (see also [CAS-2])) of a left ideal of a Weyl algebra is a Gröbner $\delta$-base with respect to an appropriate well-ordering. We also prove that the converse is not true.

We can deduce adapted algorithms for membership problem, elimination problem and syzygies problem by using Gröbner $\delta$-bases (instead of Gröbner bases) that could have in some cases lower complexity (see Remark 28).

In section 7 we apply previous results to the study of flatness of some modules in a (local) relative situation. We also give a finiteness results for some modules. These flatness results could be compared to those of [SAB] for Rees modules over Rees rings.

It is a pleasure to thank Professor A. Assi for his help and useful suggestions. We thank the referee for his useful comments.

2. Gröbner $\delta$-bases. Here, $\mathbf{k}$ is a field of characteristic 0 . Let us denote by $\mathbf{k}[[X]]=$ $\mathbf{k}\left[\left[x_{1}, \ldots, x_{n}\right]\right]$ the ring of formal power series and by $\mathbf{k}((X))$ its quotient field.

Let us denote by $\mathbf{k}((X))[\partial]=\mathbf{k}((X))\left[\partial_{1}, \ldots, \partial_{n}\right]$ the ring of linear differential operators with coefficients in $\mathbf{k}((X))$, where $\partial_{i}$ stands for the partial derivative with respect to the variable $x_{i}$.

Let us consider a noetherian sub-k-algebra $\mathcal{H} \subset \mathbf{k}((X))$ stable under the action of the partial derivatives $\partial_{1}, \ldots, \partial_{n}$. Let us denote by $\mathcal{D}$ the sub-k-algebra $($ of $\mathbf{k}((X))[\partial])$ of linear differential operators generated by $\mathcal{H}$ and $\left\{\partial_{1}, \ldots, \partial_{n}\right\}$.

More generally, we will consider differential rings as $\mathcal{D}=\mathcal{H}\left[\partial_{1}, \ldots, \partial_{n}\right]$ for any noetherian sub-k-algebra $\mathcal{H}$ of $\mathbf{k}((\tilde{X}))=\mathbf{k}\left(\left(x_{1}, \ldots, x_{n}, x_{n+1}, \ldots, x_{n+m}\right)\right)$, stable under the action of $\partial_{i}$ for $i=1, \ldots, n$.

The ring $\mathcal{D}$ is the set of formal finite sums

$$
\sum_{\alpha \in \mathbf{N}^{n}} p_{\alpha} \partial^{\alpha},
$$

where $p_{\alpha} \in \mathcal{H}$.

Let $<$ be a well-ordering in $\mathbf{N}^{n}$ compatible with the sum (i.e. a well-ordering such that, for all $\gamma \in \mathbf{N}^{n}$, we have $\alpha+\gamma<\beta+\gamma$ if and only if $\alpha<\beta$ ).

Definition 1. Let $P=\sum_{\alpha \in \mathbf{N}^{n}} p_{\alpha} \partial^{\alpha}$ be a non-zero element of $\mathcal{D}$. The Newton $\delta$ diagram of $P$ is the set

$$
\mathcal{N}^{\delta}(P)=\left\{\alpha \in \mathbf{N}^{n}: p_{\alpha} \neq 0\right\} .
$$

Definition 2. Let $P$ be a non-zero element of $\mathcal{D}$. We call the element of $\mathbf{N}^{n}$, $\max _{<}\left\{\mathcal{N}^{\delta}(P)\right\}$, the $\delta$-exponent of $P$ with respect to $<$. It will be denoted by $\exp _{<}^{\delta}(P)$ or by $\exp ^{\delta}(P)$ when no confusion is possible.

Definition 3. Let $P$ be a non-zero element of $\mathcal{D}$. We call the element $p_{\alpha} \in \mathcal{H}$, where $\alpha=\exp ^{\delta}(P)$, the $\delta$-coefficient of $P$ with respect to $<$. It will be denoted by $c_{<}^{\delta}(P)$ or by $c^{\delta}(P)$ when no confusion is possible.

With these notations we have the following (see [MOR, pages 106-108]): 
Lemma 4. Given two non-zero elements $P, Q$ in $\mathcal{D}$, the following properties hold:

1. $\exp ^{\delta}(P Q)=\exp ^{\delta}(P)+\exp ^{\delta}(Q)$ and $\exp ^{\delta}([P, Q])<\exp ^{\delta}(P Q)$.

2. If $\exp ^{\delta}(P) \neq \exp ^{\delta}(Q)$ then $\exp ^{\delta}(P+Q)=\max _{<}\left\{\exp ^{\delta}(P), \exp ^{\delta}(Q)\right\}$.

3. If $\exp ^{\delta}(P)=\exp ^{\delta}(Q)$ and $c^{\delta}(P)+c^{\delta}(Q) \neq 0$ then $\exp ^{\delta}(P+Q)=\exp ^{\delta}(P)=$ $\exp ^{\delta}(Q)$ and $c^{\delta}(P+Q)=c^{\delta}(P)+c^{\delta}(Q)$.

4. If $\exp ^{\delta}(P)=\exp ^{\delta}(Q)$ and $c^{\delta}(P)+c^{\delta}(Q)=0$ then $\exp ^{\delta}(P+Q)<\exp ^{\delta}(P)$.

All ideals we will consider in $\mathcal{D}$ will be left ideals. Let $I$ be a non-zero ideal of $\mathcal{D}$. We denote by

$$
\operatorname{Exp}_{<}^{\delta}(I)=\left\{\exp ^{\delta}(P): P \in I \backslash\{0\}\right\} \subseteq \mathbf{N}^{n} .
$$

We write $\operatorname{Exp}^{\delta}(I)$ when no confusion is possible.

REMARK 5. By Lemma 4 we have $\operatorname{Exp}^{\delta}(I)+\mathbf{N}^{n}=\operatorname{Exp}^{\delta}(I)$. So, by Dickson's Lemma (see for example [CLO]), there is a finite generating subset $F$ of $\operatorname{Exp}^{\delta}(I)$, i.e.

$$
\operatorname{Exp}^{\delta}(I)=\cup_{\alpha \in F}\left(\alpha+\mathbf{N}^{n}\right) .
$$

Any of the subsets $F$ is called a $\delta$-stair of $I$.

We denote by $\mathcal{H}[\zeta]=\mathcal{H}\left[\zeta_{1}, \ldots, \zeta_{n}\right]$ the (commutative) polynomial ring with coefficients in $\mathcal{H}$ and with variables $\zeta_{1}, \ldots, \zeta_{n}$.

Definition 6. Let $P=\sum_{\alpha} p_{\alpha} \partial^{\alpha}$ be a non-zero element of $\mathcal{D}$. The $\delta$-initial form of $P$, with respect to $<$, is $i n_{<}^{\delta}(P)=c^{\delta}(P) \zeta^{\exp ^{\delta}(P)} \in \mathcal{H}[\zeta]$. We write $i n^{\delta}(P)$ when no confusion is possible.

Definition 7. Let $I$ be a non-zero ideal of $\mathcal{D}$. The ideal generated by

$$
\left\{\operatorname{in}^{\delta}(P): P \in I \backslash\{0\}\right\}
$$

is called the $\delta$-initial ideal of $I$ with respect to $<$ (and it is denoted by $\left.i n_{<}^{\delta}(I)\right)$. We write $i n^{\delta}(I)$ when no confusion is possible.

REMARK 8. Note that $i n^{\delta}(I)$ is a $\zeta$-monomial ideal in $\mathcal{H}[\zeta]$. If $I$ is generated by $\left\{P_{1}, \ldots, P_{m}\right\}$ (i.e. $\left.I=\mathcal{D}\left(P_{1}, \ldots, P_{m}\right)\right)$, then the ideals $\mathcal{H}[\zeta]\left(i n^{\delta}\left(P_{1}\right), \ldots, i n^{\delta}\left(P_{m}\right)\right)$ and $i n^{\delta}(I)$ may be different.

Definition 9. Let $I$ be a non-zero ideal of $\mathcal{D}$. A finite family $\left\{P_{1}, \ldots, P_{m}\right\} \subset I$ is called a Gröbner $\delta$-base of $I$, with respect to the well-ordering $<$, if

$$
i n^{\delta}(I)=\mathcal{H}[\zeta]\left(i n^{\delta}\left(P_{1}\right), \ldots, i n^{\delta}\left(P_{m}\right)\right) .
$$

REMARK 10. When $\mathcal{H}=\mathbf{k}$ (i.e. in the ring $\mathbf{k}[\partial]=\mathbf{k}\left[\partial_{1}, \ldots, \partial_{n}\right]$ ), the notion of Gröbner $\delta$-base and the one of Gröbner base coincide. Here $\mathbf{k}[\partial]$ is the ring of linear differential operators with constant coefficients which is a commutative polynomial ring.

3. Reduction in $\mathcal{D}$. Let $F$ be a non-empty subset of $\mathcal{D}$ and $\alpha \in \mathbf{N}^{n}$. Here we will use some notations of $[$ ASS-1]. Let

$$
K(\alpha ; F)=\left\{c^{\delta}(P): P \in F, \alpha \in \exp ^{\delta}(P)+\mathbf{N}^{n}\right\} .
$$


We denote by $C(\alpha ; F)$ the ideal, in $\mathcal{H}$, generated by $K(\alpha ; F)$, i.e.

$$
C(\alpha ; F)=\mathcal{H} K(\alpha ; F) .
$$

If $K(\alpha ; F)=\emptyset$ then $C(\alpha ; F)=\{0\}$.

EXAmple 1. Let us consider $\mathcal{H}=\mathbf{k}\left[\left[x_{1}, x_{2}\right]\right]\left[x_{1}^{-1}, x_{2}^{-1}\right]$ and $F=\left\{P_{1}, P_{2}\right\} \subseteq \mathcal{D}=$ $\mathcal{H}\left[\partial_{1}, \partial_{2}\right]$, where $P_{1}=a \partial_{1}^{2}+b \partial_{1}$ and $P_{2}=c \partial_{2}^{2}+e \partial_{2}$ with $a, e \in \mathbf{k}\left[\left[x_{1}\right]\right]\left[x_{1}^{-1}\right], a \neq 0$ and $b, c \in \mathbf{k}\left[\left[x_{2}\right]\right]\left[x_{2}^{-1}\right]$ with $c \neq 0$. We consider the lexicographic order (cf. [CLO]) in $\mathbf{N}^{2}$ with $\partial_{1}>\partial_{2}$.

Then $\exp ^{\delta}\left(P_{1}\right)=(2,0)$ and $\exp ^{\delta}\left(P_{2}\right)=(0,2)$. Since $K((1,1) ; F)=\emptyset$ we have $C((1,1) ; F)=\{0\}$. We also have

$$
C((2,2) ; F)=\langle a, c\rangle, \quad C((2,0) ; F)=\langle a\rangle, \quad C((0,2) ; F)=\langle c\rangle,
$$

where $\langle N\rangle$ stands for the ideal (in $\mathcal{H}$ ) generated by $N$.

It is easy to check that

1. If $\beta \in \alpha+\mathbf{N}^{n}$ then $K(\alpha ; F) \subseteq K(\beta ; F)$ and $C(\alpha ; F) \subseteq C(\beta ; F)$.

2. If $F_{1} \subseteq F_{2}$ then $K\left(\alpha ; F_{1}\right) \subseteq K\left(\alpha ; F_{2}\right)$ and $C\left(\alpha ; F_{1}\right) \subseteq C\left(\alpha ; F_{2}\right)$ for all $\alpha \in \mathbf{N}^{n}$.

REMARK 11. If $I$ is a non-zero ideal of $\mathcal{D}$ then:

a) $K(\alpha ; I)=\left\{c^{\delta}(P): P \in I, \exp ^{\delta}(P)=\alpha\right\}$. Moreover, $K(\alpha ; I) \bigcup\{0\}=C(\alpha ; I)$.

b) $C(0 ; I)=I \cap \mathcal{H}$.

REMARK 12. From now on, as in [IN-PA], we suppose $\mathcal{H}$ satisfies two additional conditions:

1) For any subset $\left\{f_{1}, \ldots, f_{r}\right\} \subset \mathcal{H}$ and for any $f \in \mathcal{H}$ we can decide if $f \in \mathcal{H}\left(f_{1}, \ldots, f_{r}\right)$, and in this case, it is possible to find $q_{1}, \ldots, q_{r} \in \mathcal{H}$ such that $f=\sum_{i=1}^{r} q_{i} f_{i}$.

2) For any subset $\left\{f_{1}, \ldots, f_{r}\right\} \subset \mathcal{H}$ it is possible to find a system of generators of the $\mathcal{H}$-module of syzygies of $\left\{f_{1}, \ldots, f_{r}\right\}$.

The algebras

$$
\mathcal{H}=\mathbf{k}[X], \mathbf{k}[X]\left[x_{1}^{-1}, \ldots, x_{n}^{-1}\right], \mathbf{k}(X), \mathbf{k}[[X]], \mathbf{k}((X)), \mathbf{k}[[X]]\left[x_{1}^{-1}, \ldots, x_{n}^{-1}\right]
$$

and the algebra $\mathbf{k}\{X\}\left[x_{1}^{-1}, \ldots, x_{n}^{-1}\right]$ with $\mathbf{k}=\mathbf{R}$ or $\mathbf{C}$ satisfy conditions 1$)$ and 2$)$. See [TRI] and [IN-PA] for more details about conditions 1 . and 2.

Definition 13. Let $F=\left\{P_{1}, \ldots, P_{m}\right\} \subseteq \mathcal{D}$ with $P_{i} \neq 0,1 \leq i \leq m$, and let $P \in \mathcal{D}$. We will say that $P$ is reduced with respect to $F$ if one of the following conditions holds:

- $\exp ^{\delta}(P) \notin \bigcup_{i=1}^{m}\left(\exp ^{\delta}\left(P_{i}\right)+\mathbf{N}^{n}\right)$

- $\exp ^{\delta}(P) \in \bigcup_{i=1}^{m}\left(\exp ^{\delta}\left(P_{i}\right)+\mathbf{N}^{n}\right)$ and $c^{\delta}(P) \notin C\left(\exp ^{\delta}(P) ; F\right)$.

Let $F$ be a non-empty subset of $\mathcal{D} \backslash\{0\}$. We denote

$$
R(F)=\{R \in \mathcal{D}: R \text { is reduced with respect to } F\} .
$$

REMARK 14. $R(F)$ is not necessarily a vector space over $\mathbf{k}$.

Theorem 15 (Reduction algorithm). Let $F=\left\{P_{1}, \ldots, P_{m}\right\} \subseteq \mathcal{D}$, with $P_{i} \neq 0, i=$ $1, \ldots, m$, and $P \in \mathcal{D}$. Then there exist $Q_{1}, \ldots, Q_{m}, R \in \mathcal{D}$ such that

1. $P=\sum_{i=1}^{m} Q_{i} P_{i}+R$. 
2. $R \in R(F)$.

3. $\max _{1 \leq i \leq m}\left\{\exp ^{\delta}\left(Q_{i} P_{i}\right), \exp ^{\delta}(R)\right\}=\exp ^{\delta}(P)$.

Proof. We proceed by induction on $\exp ^{\delta}(P)=\alpha$.

If $\alpha=0$, then $P \in \mathcal{H}$. So, we can consider two cases:

1. If $0 \notin \bigcup_{i=1}^{m}\left(\exp ^{\delta}\left(P_{i}\right)+\mathbf{N}^{n}\right)$, then

$$
P=\sum_{i=1}^{m} 0 P_{i}+P, \quad \text { with } \quad P \in R(F) .
$$

2. If $0 \in \bigcup_{i=1}^{m}\left(\exp ^{\delta}\left(P_{i}\right)+\mathbf{N}^{n}\right)$, then we consider the set

$$
\Lambda=\left\{i: 0 \in \exp ^{\delta}\left(P_{i}\right)+\mathbf{N}^{n}\right\}=\left\{i: \exp ^{\delta}\left(P_{i}\right)=0\right\} .
$$

Thus for $i \in \Lambda$ we have $P_{i} \in \mathcal{H}$ and we can consider two cases:

(a) If $P \in \mathcal{H}\left(P_{i}: i \in \Lambda\right)$ then $P=\sum_{i \in \Lambda} q_{i} P_{i}$ with $q_{i} \in \mathcal{H}$ (according to our assumption on $\mathcal{H}$ we can calculate such elements $q_{i}$ ). In this case we have $P=\sum Q_{i} P_{i}+R$ where $Q_{i}=q_{i}$, for $i \in \Lambda ; Q_{i}=0$ for $i \notin \Lambda$ and $R=0$.

(b) If $P \notin \mathcal{H}\left(P_{i}: i \in \Lambda\right)$ then $P \in R(F)$.

Suppose $\alpha>0$ and the theorem is proved for $\exp ^{\delta}(P)<\alpha$.

Let $P \in \mathcal{D}$ be such that $\exp ^{\delta}(P)=\alpha$. We have two possible cases:

1. If $\alpha \notin \bigcup_{i=1}^{m}\left(\exp ^{\delta}\left(P_{i}\right)+\mathbf{N}^{n}\right)$, then $P=\sum_{i=1}^{m} 0 P_{i}+P$ and $P \in R(F)$.

2. If $\alpha \in \bigcup_{i=1}^{m}\left(\exp ^{\delta}\left(P_{i}\right)+\mathbf{N}^{n}\right)$ then we consider the set

$$
\Lambda=\left\{i: \alpha \in \exp ^{\delta}\left(P_{i}\right)+\mathbf{N}^{n}\right\}
$$

and the following two cases are possible:

(a) If $c^{\delta}(P) \in C(\alpha ; F)$, then there exists $\left(q_{i}\right)_{i \in \Lambda} \in \mathcal{H}$ such that

$$
c^{\delta}(P)=\sum_{i \in \Lambda} q_{i} c^{\delta}\left(P_{i}\right)
$$

We may write

$$
P^{(1)}=P-\sum_{i \in \Lambda} q_{i} \partial^{\gamma^{i}} P_{i}, \quad \text { with } \quad \gamma^{i}+\exp ^{\delta}\left(P_{i}\right)=\alpha .
$$

By construction, $\exp ^{\delta}\left(P^{(1)}\right)<\exp ^{\delta}(P)$. Hence, by induction, we may write $P^{(1)}=\sum_{i=1}^{m} Q_{i}^{\prime} P_{i}+R^{\prime}$, with $R^{\prime} \in R(F)$ and finally $P=\sum_{i \notin \Lambda} Q_{i}^{\prime} P_{i}+$ $\sum_{i \in \Lambda}\left(Q_{i}^{\prime}+q_{i} \partial^{\gamma^{i}}\right) P_{i}+R^{\prime}$

(b) If $c^{\delta}(P) \notin C(\alpha ; F)$ then $P \in R(F)$.

So, we have proved the existence of $Q_{1}, \ldots, Q_{m}, R$ satisfying conditions 1 . and 2. of the statement. Condition 3. is easy to verify. That ends the proof.

REMARK 16. We call $R \in \mathcal{D}$ a remainder of the reduction of $P$ by $\left(P_{1}, \ldots, P_{m}\right) \in$ $\mathcal{D}^{m}$. We denote by $\widetilde{R}\left(P ; P_{1}, \ldots, P_{m}\right)$ the set of remainders of the reduction of $P$ by $\left\{P_{1}, \ldots, P_{m}\right\}$.

REMARK 17. The proof of Theorem 15 provides an algorithm to reduce an operator $P \in \mathcal{D}$ with respect to a subset $F$ of $\mathcal{D}$. 
TheOREM 18. Let I be a non-zero ideal of $\mathcal{D}$ and $\left\{P_{1}, \ldots, P_{r}\right\} \subset I$. Then the following statements are equivalent:

1. $\left\{P_{1}, \ldots, P_{r}\right\}$ is a Gröbner $\delta$-base of $I$.

2. For $\alpha \in \mathbf{N}^{n}$, we have $C(\alpha ; I)=C\left(\alpha ; P_{1}, \ldots, P_{r}\right)$.

3. For $P \in I$ we have $\widetilde{R}\left(P ; P_{1}, \ldots, P_{r}\right)=\{0\}$.

Proof. 1. $\Rightarrow 2 .: C\left(\alpha ; P_{1}, \ldots, P_{r}\right)$ is clearly contained in $C(\alpha ; I)$. Conversely, let $p(x) \in$ $C(\alpha ; I)$ then there exists $P \in I \backslash\{0\}$ such that $i n^{\delta}(P)=p(x) \zeta^{\alpha}$ and $i n^{\delta}(P) \in i n^{\delta}(I)$. But by hypothesis, we have

$$
i n^{\delta}(I)=\mathcal{H}[\zeta]\left(i n^{\delta}\left(P_{1}\right), \ldots, i n^{\delta}\left(P_{r}\right)\right) .
$$

Let us denote

$$
\operatorname{in}^{\delta}\left(P_{i}\right)=p_{i}(x) \zeta^{\alpha_{i}} \quad \text { with } \quad 1 \leq i \leq r
$$

then

$$
p(x) \zeta^{\alpha}=\sum_{i=1}^{r} q_{i}(x, \zeta) p_{i}(x) \zeta^{\alpha_{i}},
$$

where

$$
q_{i}(x, \zeta)=\sum_{\beta} q_{i_{\beta}}(x) \zeta^{\beta} \in \mathcal{H}[\zeta] \quad \text { with } \quad q_{i_{\beta}}(x) \in \mathcal{H}
$$

Thus,

$$
p(x) \zeta^{\alpha}=\sum_{i, \beta} q_{i_{\beta}}(x) p_{i}(x) \zeta^{\beta+\alpha_{i}}
$$

hence,

$$
p(x) \zeta^{\alpha} \in \mathcal{H}[\zeta]\left(p_{i}(x) \zeta^{\alpha_{i}}: \alpha \in \alpha_{i}+\mathbf{N}^{n}\right)
$$

and so,

$$
p(x) \in C\left(\alpha ; P_{1}, \ldots, P_{r}\right) .
$$

Therefore $C(\alpha ; I) \subseteq C\left(\alpha ; P_{1}, \ldots, P_{r}\right)$ and it follows that

$$
C(\alpha ; I)=C\left(\alpha ; P_{1}, \ldots, P_{r}\right) .
$$

2. $\Rightarrow$ 3.: Let $P \in I \backslash\{0\}$, then by Theorem 15 , there exist $Q_{1}, \ldots, Q_{r}, R \in \mathcal{D}$ such that

$$
P=\sum_{i=1}^{r} Q_{i} P_{i}+R,
$$

where $R \in \widetilde{R}\left(P ; P_{1}, \ldots, P_{r}\right)$.

Suppose $R \neq 0$. Since $R=P-\sum_{i=1}^{r} Q_{i} P_{i} \in I$, we can consider two cases:

i) If $\exp ^{\delta}(R) \notin \bigcup_{i=1}^{r}\left(\exp ^{\delta}\left(P_{i}\right)+\mathbf{N}^{n}\right)$, then $C\left(\exp ^{\delta}(R) ; P_{1}, \ldots, P_{r}\right)=(0)$. Therefore $c^{\delta}(R) \notin C\left(\exp ^{\delta}(R) ; P_{1}, \ldots, P_{r}\right)$ and by hypothesis $2, c^{\delta}(R) \notin C\left(\exp ^{\delta}(R) ; I\right)$. But this is impossible since $R \in I$.

ii) If $\exp ^{\delta}(R) \in \bigcup_{i=1}^{r}\left(\exp ^{\delta}\left(P_{i}\right)+\mathbf{N}^{n}\right)$, then

$$
c^{\delta}(R) \notin C\left(\exp ^{\delta}(R) ; P_{1}, \ldots, P_{r}\right)=C\left(\exp ^{\delta}(R) ; I\right)
$$

because $R$ is reduced with respect to $\left\{P_{1}, \ldots, P_{r}\right\}$, and this contradicts that $R \in I$. Therefore $R=0$. 
3. $\Rightarrow 1 .:$ We must show that $i n^{\delta}(I)=\mathcal{H}[\zeta]\left(i n^{\delta}\left(P_{1}\right), \ldots, i n^{\delta}\left(P_{r}\right)\right)$. Clearly $\left\langle i n^{\delta}\left(P_{1}\right), \ldots\right.$, $\left.i n^{\delta}\left(P_{r}\right)\right\rangle \subseteq i n^{\delta}(I)$. Let $P \in I$ be a non-zero operator. Then we can write

$$
P=p_{\alpha_{0}} \partial^{\alpha_{0}}+\widehat{P}
$$

where $p_{\alpha_{0}} \in \mathcal{H} \backslash\{0\}$ and $\exp ^{\delta}(\widehat{P})<\alpha_{0}$.

Then, by hypothesis and by Theorem 15 , we have:

$$
\alpha_{0} \in \bigcup_{i=1}^{r}\left(\exp ^{\delta}\left(P_{i}\right)+\mathbf{N}^{n}\right) \quad \text { and } \quad c^{\delta}(P) \in C\left(\alpha_{0} ; P_{1}, \ldots, P_{r}\right) .
$$

We consider the set $\Lambda=\left\{i: \alpha_{0} \in \exp ^{\delta}\left(P_{i}\right)+\mathbf{N}^{n}\right\}$. Then $c^{\delta}(P)=\sum_{i \in \Lambda} q_{i}^{(1)} c^{\delta}\left(P_{i}\right)$.

Let

$$
P^{(1)}=P-\sum_{i \in \Lambda} q_{i}^{(1)} \partial^{\gamma^{i}} P_{i}, \quad \text { with } \quad \gamma^{i}+\exp ^{\delta}\left(P_{i}\right)=\alpha_{0},
$$

then $P^{(1)} \in I$ and $\exp ^{\delta}\left(P^{(1)}\right)<\alpha_{0}$.

Now we can consider two cases:

i) If $P^{(1)}=0$, then $P=\sum_{i \in \Lambda} q_{i}^{(1)} \partial^{\gamma^{i}} P_{i}$ and it can be checked that

$$
i n^{\delta}(P)=\sum_{i \in \Lambda} q_{i}^{(1)} \zeta^{\gamma^{i}} i n^{\delta}\left(P_{i}\right)
$$

ii) If $P^{(1)} \neq 0$, then by repeating the same procedure, we can obtain a family $P^{(k)} \in I$ with $\exp ^{\delta}\left(P^{(k)}\right)<\exp ^{\delta}\left(P^{(k-1)}\right)$. So, as $<$ is a well-ordering in $\mathbf{N}^{n}$, there exists $l$ such that $P^{(l)}=0$.

This completes the proof.

As a straightforward consequence of Theorem 18 we get the following result:

COROLlary 19. Any Gröbner $\delta$-base of an ideal $I \subseteq \mathcal{D}$ is a system of generators of I. Moreover, if $\left\{P_{1}, \ldots, P_{r}\right\}$ is a Gröbner $\delta$-base of $I$ then

$$
\operatorname{Exp}^{\delta}(I)=\bigcup_{i=1}^{r}\left(\exp ^{\delta}\left(P_{i}\right)+\mathbf{N}^{n}\right) .
$$

4. $S^{\delta}$-operators. Let $F=\left\{P_{1}, \ldots, P_{r}\right\} \subseteq \mathcal{D} \backslash\{0\}$. Let

$$
K(F)=\left\{\alpha \in \mathbf{N}^{n}: \exists N \subseteq F, \alpha=l c m\left\{\exp ^{\delta}(P) ; P \in N\right\}\right\},
$$

where $l \mathrm{~cm}$ stands for least common multiple, and $F_{\alpha}=\left\{\left(\lambda_{1}, \ldots, \lambda_{r}\right) \in \mathcal{H}^{r}: \sum_{k=1}^{r} \lambda_{k} c^{\delta}\left(P_{k}\right)=0\right.$ where $\lambda_{k}=0$ if $\left.\alpha \notin \exp ^{\delta}\left(P_{k}\right)+\mathbf{N}^{n}\right\} \subseteq \mathcal{H}^{r}$ $F_{\alpha}$ is isomorphic to the $\mathcal{H}$-module of syzygies of

$$
\left\{c^{\delta}\left(P_{k}\right): \alpha \in \exp ^{\delta}\left(P_{k}\right)+\mathbf{N}^{n}, 1 \leq k \leq r\right\} .
$$

Since $\mathcal{H}$ is a noetherian algebra $F_{\alpha}$ is finitely generated (as an $\mathcal{H}$-module). Let $\left\{\left(\lambda_{1}^{\tau}, \ldots, \lambda_{r}^{\tau}\right)\right\}, 1 \leq \tau \leq r_{\alpha}$, be a system of generators of $F_{\alpha}$. 
Definition 20. With the notations as above, for $\tau=1, \ldots, r_{\alpha}$, the element

$$
S_{\alpha, \tau}^{\delta}=\sum_{k=1}^{r} \lambda_{k}^{\tau} \partial^{\alpha-\exp ^{\delta}\left(P_{k}\right)} P_{k}
$$

will be called an $S^{\delta}$-operator of the set $F_{\alpha}$.

Proposition 21. With the notations as above, we have

$$
\exp ^{\delta}\left(S_{\alpha, \tau}^{\delta}\right)<\alpha
$$

Proof. We can write

$$
\begin{gathered}
S_{\alpha, \tau}^{\delta}=\sum_{k=1}^{r} \lambda_{k}^{\tau} \partial^{\alpha-\exp ^{\delta}\left(P_{k}\right)} P_{k} \\
=\sum_{k=1}^{r} \lambda_{k}^{\tau} \partial^{\alpha-\exp ^{\delta}\left(P_{k}\right)} c^{\delta}\left(P_{k}\right) \partial^{\exp ^{\delta}\left(P_{k}\right)}+\sum_{k=1}^{r} \sum_{\beta<\exp ^{\delta}\left(P_{k}\right)} \lambda_{k}^{\tau} \partial^{\alpha-\exp ^{\delta}\left(P_{k}\right)} p_{\beta, k} \partial^{\beta} .
\end{gathered}
$$

Since

$$
\begin{aligned}
\partial^{\alpha-\exp ^{\delta}\left(P_{k}\right)} c^{\delta}\left(P_{k}\right) & =c^{\delta}\left(P_{k}\right) \partial^{\alpha-\exp ^{\delta}\left(P_{k}\right)}+A_{k} \quad \text { with } \quad \exp ^{\delta}\left(A_{k}\right)<\alpha-\exp ^{\delta}\left(P_{k}\right), \\
\partial^{\alpha-\exp ^{\delta}\left(P_{k}\right)} p_{\beta, k} & =p_{\beta, k} \partial^{\alpha-\exp ^{\delta}\left(P_{k}\right)}+B_{k} \quad \text { with } \quad \exp ^{\delta}\left(B_{k}\right)<\alpha-\exp ^{\delta}\left(P_{k}\right)
\end{aligned}
$$

and $\sum_{k=1}^{r} \lambda_{k}^{\tau} c^{\delta}\left(P_{k}\right)=0$, finally

$$
S_{\alpha, \tau}^{\delta}=\sum_{k=1}^{r} \lambda_{k}^{\tau} A_{k} \partial^{\exp ^{\delta}\left(P_{k}\right)}+\sum_{k=1}^{r} \sum_{\beta<\exp ^{\delta}\left(P_{k}\right)}\left(\lambda_{k}^{\tau} p_{\beta, k} \partial^{\alpha-\exp ^{\delta}\left(P_{k}\right)+\beta}+B_{k} \partial^{\beta}\right)
$$

Proposition 22. Let $I$ be a non-zero ideal of $\mathcal{D}$ and $\left\{P_{1}, \ldots, P_{r}\right\}$ be a system of generators of $I$. Then the following are equivalent:

1. $\left\{P_{1}, \ldots, P_{r}\right\}$ is a $\delta$-Gröbner base of $I$.

2. For all $P \in I$, we have $\widetilde{R}\left(P ; P_{1}, \ldots, P_{r}\right)=\{0\}$.

3. For every $S^{\delta}$-operator, $S_{\alpha, \tau}^{\delta}$, of $\left\{P_{1}, \ldots, P_{r}\right\}$ we have $0 \in \widetilde{R}\left(S_{\alpha, \tau}^{\delta} ; P_{1}, \ldots, P_{r}\right)$.

Proof. We know, by Theorem 18, the equivalence between 1. and 2.

2. $\Rightarrow 3$.: Since $S_{\alpha, \tau}^{\delta} \in I$, then, by assumption, $0 \in \widetilde{R}\left(S_{\alpha, \tau}^{\delta} ; P_{1}, \ldots, P_{r}\right)$.

3. $\Rightarrow$ 1.: Let $P \in I$ be a non-zero operator. We must show that $\operatorname{in}^{\delta}(P) \in$ $\mathcal{H}[\zeta]\left(i n^{\delta}\left(P_{1}\right), \ldots, i n^{\delta}\left(P_{r}\right)\right)$. We may write $P=\sum_{i=1}^{r} H_{i} P_{i}$, with $H_{i} \in \mathcal{H}[\partial]$.

Suppose

$$
\alpha_{0}=\max _{i}\left\{\exp ^{\delta}\left(H_{i} P_{i}\right)\right\} \quad \text { and } \quad \exp ^{\delta}\left(H_{i_{k}} P_{i_{k}}\right)=\alpha_{0}, \quad k=0, \ldots, t .
$$

Hence, by Lemma 4,

$$
\exp ^{\delta}\left(H_{i_{k}}\right)+\exp ^{\delta}\left(P_{i_{k}}\right)=\alpha_{0}, \quad k=0, \ldots, t .
$$

We can consider two cases:

a) If $\sum_{k=0}^{t} c^{\delta}\left(H_{i_{k}}\right) c^{\delta}\left(P_{i_{k}}\right) \neq 0$ then

$$
i n^{\delta}(P)=c^{\delta}(P) \zeta^{\alpha_{0}}, \text { with } c^{\delta}(P)=\sum_{k=0}^{t} c^{\delta}\left(H_{i_{k}}\right) c^{\delta}\left(P_{i_{k}}\right) .
$$


Therefore,

$$
i n^{\delta}(P)=\sum_{k=0}^{t} c^{\delta}\left(H_{i_{k}}\right) c^{\delta}\left(P_{i_{k}}\right) \zeta^{\alpha_{0}}=\sum_{k=0}^{t} c^{\delta}\left(H_{i_{k}}\right) \zeta^{\alpha_{0}-\exp ^{\delta}\left(P_{i_{k}}\right)} i n^{\delta}\left(P_{i_{k}}\right)
$$

and so, $i n^{\delta}(P) \in \mathcal{H}[\zeta]\left(i n^{\delta}\left(P_{1}\right), \ldots, i n^{\delta}\left(P_{r}\right)\right)$.

b) Suppose now $\sum_{k=0}^{t} c^{\delta}\left(H_{i_{k}}\right) c^{\delta}\left(P_{i_{k}} t\right)=0$. Let us denote $\alpha^{i}=\exp ^{\delta}\left(P_{i}\right), i=1, \ldots, r$; we consider the set $\Lambda=\left\{i: \exp ^{\delta}\left(H_{i}\right)+\alpha^{i}=\alpha_{0}\right\}$, and we suppose $\gamma=l \operatorname{cm}\left\{\exp ^{\delta}\left(P_{i}\right)\right.$ : $i \in \Lambda$ \}. We may write

$$
P=\sum_{i \notin \Lambda} H_{i} P_{i}+\sum_{i \in \Lambda} c^{\delta}\left(H_{i}\right) \partial^{\exp ^{\delta}\left(H_{i}\right)} P_{i}+\sum_{i \in \Lambda}\left(H_{i}-c^{\delta}\left(H_{i}\right) \partial^{\exp ^{\delta}\left(H_{i}\right)}\right) P_{i} .
$$

We can identify $\left(c^{\delta}\left(H_{i}\right)\right)_{i \in \Lambda}$ with an element of $F_{\gamma}$. Let $\underline{\lambda}^{1}, \ldots, \underline{\lambda}^{p}$ be a family of generators of $F_{\gamma}$ where

$$
\underline{\lambda}^{\tau}=\left(\lambda_{1}^{\tau}, \ldots, \lambda_{r}^{\tau}\right) \quad \text { with } \quad \lambda_{j}^{\tau}=0 \quad \text { if } \quad \gamma \notin \exp ^{\delta}\left(P_{j}\right)+\mathbf{N}^{n} .
$$

Now for each $i \in\{1, \ldots, r\}$ we define $s_{i}$ as follows:

$$
s_{i}=\left\{\begin{array}{lll}
c^{\delta}\left(H_{i}\right) & \text { if } & i \in \Lambda \\
0 & \text { if } & i \notin \Lambda .
\end{array}\right.
$$

Hence $\underline{s}=\left(s_{1}, \ldots, s_{r}\right) \in F_{\gamma}$, and then there exist $u_{1}, \ldots, u_{p} \in \mathcal{H}$ such that $\underline{s}=$ $\sum_{\tau=1}^{p} u_{\tau} \underline{\lambda}^{\tau}$. Let us denote $\beta^{i}=\exp ^{\delta}\left(H_{i}\right)$, for $i \in \Lambda$, then

$$
\sum_{i \in \Lambda} c^{\delta}\left(H_{i}\right) \partial^{\beta^{i}} P_{i}=\sum_{\tau=1}^{p} u_{\tau}\left(\sum_{i=1}^{r} \lambda_{i}^{\tau} \partial^{\beta^{i}} P_{i}\right)
$$

The element $\alpha_{0}$ is, by definition, a common multiple of the elements $\left\{\exp ^{\delta}\left(P_{i}\right)\right.$ : $i \in \Lambda$ \} then there exists $\epsilon \in \mathbf{N}^{n}$ such that $\alpha_{0}=\gamma+\epsilon$ and so $\beta^{i}=\gamma-\alpha^{i}+\epsilon$. If $j \notin \Lambda$ and $\gamma \in \exp ^{\delta}\left(P_{j}\right)+\mathbf{N}^{n}$ we denote $\beta^{j}=\gamma-\alpha^{j}+\epsilon$. Therefore,

$$
\sum_{i \in \Lambda} c^{\delta}\left(H_{i}\right) \partial^{\beta^{i}} P_{i}=\sum_{\tau=1}^{p} u_{\tau}\left(\sum_{i=1}^{r} \partial^{\epsilon} \lambda_{i}^{\tau} \partial^{\gamma-\alpha^{i}} P_{i}\right)+\sum_{\tau=1}^{p} u_{\tau}\left(\sum_{i \mid \gamma-\alpha^{i}>0} B_{i}^{\tau} \partial^{\gamma-\alpha^{i}} P_{i}\right)
$$

where $\exp ^{\delta}\left(B_{i}^{\tau}\right)<\epsilon$. Therefore, by Definition 20,

$$
\sum_{i \in \Lambda} c^{\delta}\left(H_{i}\right) \partial^{\beta^{i}} P_{i}=\sum_{\tau=1}^{p} u_{\tau} \partial^{\epsilon} S_{\gamma, \tau}^{\delta}+\sum_{i \mid \gamma-\alpha^{i}>0}\left(\sum_{\tau=1}^{p} u_{\tau} B_{i}^{\tau}\right) \partial^{\gamma-\alpha^{i}} P_{i} .
$$

But by hypothesis, we have

$$
S_{\gamma, \tau}^{\delta}=\sum_{j=1}^{r} Q_{j}^{\gamma, \tau} P_{j}
$$

with $\gamma>\exp ^{\delta}\left(S_{\gamma, \tau}^{\delta}\right)=\max _{1 \leq j \leq r}\left\{\exp ^{\delta}\left(Q_{j}^{\gamma, \tau} P_{j}\right)\right\}$. Hence,

$$
\sum_{i \in \Lambda} c^{\delta}\left(H_{i}\right) \partial^{\beta^{i}} P_{i}=\sum_{j=1}^{r}\left(\sum_{\tau=1}^{p} u_{\tau} \partial^{\epsilon} Q_{j}^{\gamma, \tau}\right) P_{j}+\sum_{j \mid \gamma-\alpha^{j}>0}\left(\sum_{\tau=1}^{p} u_{\tau} B_{j}^{\tau}\right) \partial^{\gamma-\alpha^{j}} P_{j} .
$$


Therefore

$$
P=\sum_{i=1}^{r} H_{i}^{\prime} P_{i}
$$

where

- If $i \in \Lambda$,

$$
H_{i}^{\prime}=H_{i}-c^{\delta}\left(H_{i}\right) \partial^{\beta^{i}}+\sum_{\tau=1}^{p} u_{\tau} \partial^{\epsilon} Q_{i}^{\gamma, \tau}+\sum_{\tau=1}^{p} u_{\tau} B_{i}^{\tau} \partial^{\gamma-\alpha^{i}} .
$$

- If $i \notin \Lambda$ and $\gamma-\alpha^{i}>0$,

$$
H_{i}^{\prime}=H_{i}+\sum_{\tau=1}^{p} u_{\tau} \partial^{\epsilon} Q_{i}^{\gamma, \tau}+\sum_{\tau=1}^{p} u_{\tau} B_{i}^{\tau} \partial^{\gamma-\alpha^{i}} .
$$

- If $i \notin \Lambda$ and $\gamma-\alpha^{i}$ is not greater than 0 ,

$$
H_{i}^{\prime}=H_{i}+\sum_{\tau=1}^{p} u_{\tau} \partial^{\epsilon} Q_{i}^{\gamma, \tau}
$$

Hence, we have obtained an expression for $P$ as a combination of the $P_{i}$ where $\exp ^{\delta}\left(H_{i}^{\prime} P_{i}\right)<\alpha_{0}$, then $\max _{i}\left\{\exp ^{\delta}\left(H_{i}^{\prime} P_{i}\right)\right\}<\alpha_{0}$. But this process stops because $<$ is a well-ordering in $\mathbf{N}^{n}$. So, there exists an expression of $P$ with the conditions of case a).

5. Construction of a Gröbner $\delta$-base. Let $I$ be a non-zero ideal of $\mathcal{D}$ and let $F=\left\{P_{1}, \ldots, P_{r}\right\}$ be a system of generators of $I$. We will show here how to build a Gröbner $\delta$-base of the ideal $I$ (with respect to an ordering $<$ ). We will follow the main lines of Buchberger's algorithm, adapted to our case (see [BUCH], [TRI] and [ASS-1]).

Let $K(F)=\left\{\alpha^{1}, \ldots, \alpha^{s}\right\}$ (see Section 4). Let $\left\{S_{\alpha^{j}, \tau}^{\delta}\right\}, 1 \leq j \leq s, 1 \leq \tau \leq r_{j}$ be the family of $S^{\delta}$-operators associated to $F$.

We suppose that $\left\{P_{1}, \ldots, P_{r}\right\}$ is not a Gröbner $\delta$-base for $I$, then (by Proposition 22) there exists $S_{\alpha_{0}, \tau}^{\delta}$ such that $0 \notin \widetilde{R}\left(S_{\alpha_{0}, \tau}^{\delta} ; P_{1}, \ldots, P_{r}\right)$, then let

$$
P_{r+1} \in \widetilde{R}\left(S_{\alpha_{0}, \tau}^{\delta} ; P_{1}, \ldots, P_{r}\right)
$$

and repeat this process with $\left\{P_{1}, \ldots, P_{r}, P_{r+1}\right\}$.

REMARK 23. If a $S^{\delta}$-operator, $S$, of $F$ satisfy that $0 \in \widetilde{R}\left(S ; P_{1}, \ldots, P_{r}\right)$ then $0 \in$ $\widetilde{R}\left(S ; P_{1}, \ldots, P_{r}, P_{r+1}\right)$.

The following Proposition assures that this procedure terminates.

Proposition 24. With the notations as above, there exists $\rho \in \mathbf{N}$ such that for all $S^{\delta}$-operator $S$ of $\left\{P_{1}, \ldots, P_{r+\rho}\right\}$ we have $0 \in \widetilde{R}\left(S ; P_{1}, \ldots, P_{r+\rho}\right)$.

Proof. See [MOR, pages 131-133].

6. Gröbner bases and Gröbner $\delta$-bases. In this section we will first work on the Weyl algebra $A_{n}(\mathbf{k})=\mathbf{k}[X][\partial]$, so we suppose for the moment $\mathcal{H}=\mathbf{k}[X]=\mathbf{k}\left[x_{1}, \ldots, x_{n}\right]$.

Let $<_{x},<_{\partial}$ be monomial orderings in $\mathbf{N}^{n}$. 
We denote by $X^{\alpha} \partial^{\beta}$ the monomial

$$
x_{1}^{\alpha_{1}} \ldots x_{n}^{\alpha_{n}} \partial_{1}^{\beta_{1}} \ldots \partial_{n}^{\beta_{n}} .
$$

Let us define on $\mathbf{N}^{n} \times \mathbf{N}^{n}$ the total ordering (denoted $<$ ) by

$$
(\alpha(1), \beta(1))<(\alpha(2), \beta(2)) \Leftrightarrow\left\{\begin{array}{l}
\beta(1)<_{\partial} \beta(2) \\
\text { or } \\
\beta(1)=\beta(2) \text { and } \alpha(1)<_{x} \alpha(2) .
\end{array}\right.
$$

REMARK 25. The relation $<$, defined in $\mathbf{N}^{n} \times \mathbf{N}^{n}$, is a monomial ordering. This well-ordering is called an elimination order (see for example [CLO]).

For the notion of Gröbner base on $A_{n}(\mathbf{k})$ and some related results we follow here [CAS-1] (see also [CAS-2]).

Theorem 26. Let $G=\left\{P_{1}, \ldots, P_{r}\right\}$ be a system of generators for a non-zero ideal $I \subset A_{n}(\mathbf{k})$. If $G$ is a Gröbner base for $I$, with respect to $<$, then $G$ is a Gröbner $\delta$-base for $I$ with respect to $<_{\partial}$.

Proof. Let $P \in I$ be a non-zero operator. We must show that

$$
i n^{\delta}(P) \in \mathcal{H}[\zeta]\left(i n^{\delta}\left(P_{1}\right), \ldots, i n^{\delta}\left(P_{r}\right)\right) .
$$

For $i=1, \ldots, r$, we may write $P_{i}=a_{i} \partial^{\alpha_{i}}+\widehat{P}_{i}$ where $\exp ^{\delta}\left(P_{i}\right)=\alpha_{i}, \exp ^{\delta}\left(\widehat{P}_{i}\right)<\alpha_{i}$ and $a_{i} \in \mathcal{H}$. Thus $i n^{\delta}\left(P_{i}\right)=a_{i} \zeta^{\alpha_{i}}$. By the division algorithm in $A_{n}(\mathbf{k})$, (see [CAS-1] and [CAS-2]) there exist $Q_{i_{1}}, \ldots, Q_{i_{N}} \in A_{n}(\mathbf{k}), 1 \leq i_{j} \leq r$, satisfying $P=Q_{i_{1}} P_{i_{1}}+\ldots+$ $Q_{i_{N}} P_{i_{N}}$ where $\exp _{<}\left(Q_{i} P_{i}\right) \neq \exp _{<}\left(Q_{j} P_{j}\right)$ for $i \neq j$.

We can suppose

$$
\exp _{<}\left(Q_{i_{N}} P_{i_{N}}\right)<\exp _{<}\left(Q_{i_{N-1}} P_{i_{N-1}}\right)<\ldots<\exp _{<}\left(Q_{i_{1}} P_{i_{1}}\right) .
$$

We can write

$$
Q_{i_{j}}=c_{i_{j}} \partial^{\beta_{i_{j}}}+\widehat{Q}_{i_{j}}
$$

where $\exp ^{\delta}\left(Q_{i_{j}}\right)=\beta_{i_{j}}, \exp ^{\delta}\left(\widehat{Q}_{i_{j}}\right)<\beta_{i_{j}}, c_{i_{j}} \in \mathcal{H}$. Thus $\exp _{<}\left(Q_{i_{j}}\right)=\left(\exp _{<_{x}}\left(c_{i_{j}}\right), \beta_{i_{j}}\right)$.

Therefore,

$$
P=\sum_{j=1}^{N} c_{i_{j}} a_{i_{j}} \partial^{\beta_{i_{j}}+\alpha_{i_{j}}}+\sum_{j=1}^{N} c_{i_{j}} A_{i_{j}} \partial^{\alpha_{i_{j}}}+\sum_{j=1}^{N} c_{i_{j}} \partial^{\beta_{i_{j}}} \widehat{P}_{i_{j}}+\sum_{j=1}^{N} \widehat{Q}_{i_{j}} a_{i_{j}} \partial^{\alpha_{i_{j}}}+\sum_{j=1}^{N} \widehat{Q}_{i_{j}} \widehat{P}_{i_{j}}
$$

where

$$
\begin{array}{r}
\exp ^{\delta}\left(\sum_{j=1}^{N} c_{i_{j}} a_{i_{j}} \partial^{\beta_{i_{j}}+\alpha_{i_{j}}}\right) \leq \max _{1 \leq j \leq N}\left\{\beta_{i_{j}}+\alpha_{i_{j}}\right\}, \\
\exp ^{\delta}\left(\sum_{j=1}^{N} c_{i_{j}} A_{i_{j}} \partial^{\alpha_{i_{j}}}\right)<\max _{1 \leq j \leq N}\left\{\beta_{i_{j}}+\alpha_{i_{j}}\right\}, \\
\exp ^{\delta}\left(\sum_{j=1}^{N} c_{i_{j}} \partial^{\beta_{i_{j}}} \widehat{P}_{i_{j}}\right)<\max _{1 \leq j \leq N}\left\{\beta_{i_{j}}+\alpha_{i_{j}}\right\}, \\
\exp ^{\delta}\left(\sum_{j=1}^{N} \widehat{Q}_{i_{j}} a_{i_{j}} \partial^{\alpha_{i_{j}}}\right)<\max _{1 \leq j \leq N}\left\{\beta_{i_{j}}+\alpha_{i_{j}}\right\},
\end{array}
$$




$$
\exp ^{\delta}\left(\sum_{j=1}^{N} \widehat{Q}_{i_{j}} \widehat{P}_{i_{j}}\right)<\max _{1 \leq j \leq N}\left\{\beta_{i_{j}}+\alpha_{i_{j}}\right\}
$$

Let $j_{0}$ be such that

$$
\beta_{i_{j_{0}+1}}+\alpha_{i_{j_{0}+1}}<\beta_{i_{j_{0}}}+\alpha_{i_{j_{0}}}=\beta_{i_{j_{0}-1}}+\alpha_{i_{j_{0}-1}}=\ldots=\beta_{i_{1}}+\alpha_{i_{1}} .
$$

Since $\sum_{j=1}^{j_{0}} c_{i_{j}} a_{i_{j}} \neq 0$, we have $i n^{\delta}(P)=\left(\sum_{j=1}^{j_{0}} c_{i_{j}} a_{i_{j}}\right) \zeta^{\beta_{i_{1}}+\alpha_{i_{1}}}$. Therefore,

$$
i n^{\delta}(P)=\sum_{j=1}^{j_{0}} c_{i_{j}} i n^{\delta}\left(P_{i_{j}}\right) \zeta^{\beta_{i_{j}}}
$$

and so $i n^{\delta}(P) \in \mathcal{H}[\zeta]\left(i n^{\delta}\left(P_{1}\right), \ldots, i n^{\delta}\left(P_{r}\right)\right)$. This completes the proof.

The converse of the above result is not true as we show in the following example:

ExAmple 2. Let $I \subset A_{2}(\mathbf{C})=\mathbf{C}\left[x_{1}, x_{2}\right]\left[\partial_{1}, \partial_{2}\right]$ be the left ideal generated by the operators

$$
P_{1}=x_{1} \partial_{1}+a \partial_{2}+b, \quad P_{2}=\left(x_{2}-x_{1}\right) \partial_{2}-d
$$

with $a, b, d \in \mathbf{C}\left[x_{1}, x_{2}\right]$.

We will prove ${ }^{1}$ that $\left\{P_{1}, P_{2}\right\}$ is a Gröbner $\delta$-base which is not a Gröbner base of $I$, for a particular choice of the polynomials $a, b, d$.

We have

$$
\exp _{<}\left(P_{1}\right)=(1,0,1,0), \quad \exp _{<}\left(P_{2}\right)=(1,0,0,1)
$$

Then

$$
S\left(P_{1}, P_{2}\right)=\partial_{2} P_{1}+\partial_{1} P_{2}=x_{2} \partial_{1} \partial_{2}+\partial_{2} a \partial_{2}+\partial_{2} b-\partial_{1} d-\partial_{2},
$$

and then

$$
\exp _{<}\left(S\left(P_{1}, P_{2}\right)\right)=(0,1,1,1) \notin\langle(1,0,1,0),(1,0,0,1)\rangle=\left\langle\exp _{<}\left(P_{1}\right), \exp _{<}\left(P_{2}\right)\right\rangle .
$$

So, $G=\left\{P_{1}, P_{2}\right\}$ is not a Gröbner base of the ideal $I$, for any $a, b, d \in \mathbf{C}\left[x_{1}, x_{2}\right]$.

We will prove that, for some $a, b, d \in \mathbf{C}\left[x_{1}, x_{2}\right]$, the set $G=\left\{P_{1}, P_{2}\right\}$ is a Gröbner $\delta$-base of $I$.

We have

$$
\exp ^{\delta}\left(P_{1}\right)=(1,0), \quad c^{\delta}\left(P_{1}\right)=x_{1}
$$

and

$$
\exp ^{\delta}\left(P_{2}\right)=(0,1), \quad c^{\delta}\left(P_{2}\right)=x_{2}-x_{1} .
$$

We will compute the associated $S^{\delta}$-operators (see Definition 20).

As

$$
\alpha=\operatorname{lcm}((1,0),(0,1))=(1,1)
$$

we must first compute a system of generators of

$$
F_{(1,1)}\left(P_{1}, P_{2}\right)=\left\{\left(\lambda_{1}, \lambda_{2}\right) \in \mathbf{C}\left[x_{1}, x_{2}\right]: \lambda_{1} c^{\delta}\left(P_{1}\right)+\lambda_{2} c^{\delta}\left(P_{2}\right)=0\right\} .
$$

In fact we have

$$
\operatorname{Syz}\left(c^{\delta}\left(P_{1}\right), c^{\delta}\left(P_{2}\right)\right)=F_{(1,1)}\left(P_{1}, P_{2}\right)=\left\langle\left(x_{2}-x_{1},-x_{1}\right)\right\rangle
$$

\footnotetext{
${ }^{1}$ By using the degree lexicographical order with $\partial_{2}<_{\partial} \partial_{1}$ and $x_{2}<_{x} x_{1}$.
} 
and then

$$
\begin{gathered}
S_{(1,1),\left(x_{2}-x_{1},-x_{1}\right)}^{\delta}=\left(x_{2}-x_{1}\right) \partial^{(1,1)-(1,0)} P_{1}-x_{1} \partial^{(1,1)-(0,1)} P_{2} \\
=\left(x_{2}-x_{1}\right) a \partial_{2}^{2}+\left(x_{2}-x_{1}\right) \partial_{2}(a) \partial_{2}+\left(x_{2}-x_{1}\right) b \partial_{2} \\
+\left(x_{2}-x_{1}\right) \partial_{2}(b)+x_{1} \partial_{2}+x_{1} d \partial_{1}+x_{1} \partial_{1}(d) .
\end{gathered}
$$

Now we reduce $S_{(1,1),\left(x_{2}-x_{1},-x_{1}\right)}^{\delta}$ by $\left(P_{1}, P_{2}\right)$, say

$$
\begin{gathered}
S_{(1,1),\left(x_{2}-x_{1},-x_{1}\right)}^{\delta}-d P_{1}-\partial_{2} a P_{2}-b P_{2} \\
=\left(x_{2}-x_{1}\right) \partial_{2}(b)+x_{1} \partial_{2}+x_{1} \partial_{1}(d)-a \partial_{2}+a \partial_{2}(d)+\partial_{2}(a) d \\
=\left(x_{2}-x_{1}\right) \partial_{2}(b)+\left(x_{1}-a\right) \partial_{2}+x_{1} \partial_{1}(d)+\partial_{2}(a d) .
\end{gathered}
$$

Then $\left\{P_{1}, P_{2}\right\}$ is a Gröbner $\delta$-base of $I$ if $a=x_{1}, b \in \mathbf{C}\left[x_{1}\right]$ and $d$ is a polynomial in $x_{1}-x_{2}$.

REMARK 27. The preceding Example proves a little more. Let us consider $\mathcal{H}=$ $\mathbf{C}\left[x_{1}, \ldots, x_{n}\right]$ (for $n \geq 3$ ) and the ring of differential operators $\mathcal{D}=\mathcal{H}\left[\partial_{1}, \partial_{2}\right]$ (which is a sub-algebra of the Weyl algebra $\left.A_{n}(\mathbf{C})\right)$.

Let $I \subset \mathcal{D}$ be the left ideal generated by the operators

$$
P_{1}=x_{1} \partial_{1}+a \partial_{2}+b, \quad P_{2}=\left(x_{2}-x_{1}\right) \partial_{2}-d
$$

with $a, b, d \in \mathbf{C}\left[x_{1}, \ldots, x_{n}\right]$. An analogous computation to the one of Example 2 proves that $\left\{P_{1}, P_{2}\right\}$ is a Gröbner $\delta$-base of $I$ if $a=x_{1}, b \in \mathbf{C}\left[x_{1}, x_{3}, \ldots, x_{n}\right]$ and $d$ is a polynomial in $x_{1}-x_{2}$ with coefficients in $\mathbf{C}\left[x_{3}, \ldots, x_{n}\right]$.

REMARK 28. The theory of Gröbner $\delta$-bases can be extended to submodules of a finite rank free $\mathcal{D}$-module (see [MOR, Chapter 6]). As an application one can compute free resolutions of finitely presented $\mathcal{D}$-modules.

In the particular case of the ideal $I \subset \mathcal{D}=A_{2}(\mathbf{C})$ generated by $\left\{P_{1}=x_{1} \partial_{1}+x_{1} \partial_{2}+\right.$ $\left.x_{1}, P_{2}=\left(x_{1}-x_{2}\right) \partial_{2}\right\}$, which is a Gröbner $\delta$-base according to Example 2, one can prove that the syzygy $S=\left(P_{2},-P_{1}\right)$ generates the first syzygies module of $\left\{P_{1}, P_{2}\right\}$. This leads to a free resolution of the quotient module $A_{2}(\mathbf{C}) / A_{2}(\mathbf{C}) I$ :

$$
0 \rightarrow A_{2} \rightarrow A_{2}^{2} \rightarrow A_{2} \rightarrow A_{2} / A_{2} I
$$

where $A_{2}$ stands for $A_{2}(\mathbf{C})$.

Of course we can use Gröbner bases, instead of Gröbner $\delta$-bases, for the computation of syzygies and free resolutions. In the example above, that technique will produce a free resolution of length 4 where the ranks of the free $\mathcal{D}$-modules involved are $1,3,3,1$. So, one can consider that, in this case, the use of Gröbner $\delta$-bases produces a "better" free resolution, a fact which surely merits some explanation for the general case, which cannot be given here.

We can also prove an analogue to Theorem 26 for the ring $\mathcal{D}$ of linear differential operators with formal (resp. convergent) power series coefficients in $n$ variables. In this case the ring $\mathcal{H}$ will be $\mathbf{k}\left[\left[x_{1}, \ldots, x_{n}\right]\right]$ (resp. $\mathbf{k}\left\{x_{1}, \ldots, x_{n}\right\}$ for $\left.\mathbf{k}=\mathbf{R}, \mathbf{C}\right)$.

Although the result could be stated in a more general way we restrict ourselves to the case of monomial orderings (on $\mathbf{N}^{2 n}$ ) defined by linear forms (as in [CAS-1] and [CAS-2]). 
Let us denote by $<$ such an ordering on $\mathbf{N}^{2 n}$ and by $<_{\partial}$ its restriction to $\{0\} \times \mathbf{N}^{n}$. Then we have the following Theorem whose proof is similar to the one of Theorem 26 .

Theorem 29. Let $G=\left\{P_{1}, \ldots, P_{r}\right\}$ be a system of generators for a non-zero ideal $I \subset \mathcal{D}$. If $G$ is a Gröbner base for $I$, with respect to $<$, then $G$ is a Gröbner $\delta$-base for $I$ with respect to $<_{\partial}$.

The converse is not true as shown in the following example:

EXAmple 3. We can rewrite Example 2 in this new setting.

Let $I \subset \mathcal{D}=\mathbf{C}\left[\left[x_{1}, x_{2}\right]\right]\left[\partial_{1}, \partial_{2}\right]$ be the left ideal generated by the operators $P_{1}=$ $x_{1} \partial_{1}+a \partial_{2}+b, \quad P_{2}=\left(x_{2}-x_{1}\right) \partial_{2}-d$ with $a, b, d \in \mathbf{C}\left[\left[x_{1}, x_{2}\right]\right]$.

Let us consider (on $\mathbf{N}^{4}$ ) the graded lexicographic order $<$ with $x_{2}<x_{1}<\partial_{2}<\partial_{1}$. The restriction $<^{\prime}:=<_{\partial}$ on $\{0\} \times \mathbf{N}^{2}$ is then the graded lexicographic order with $\partial_{2}<\partial_{1}$.

For this particular choice of $<$, operators $P_{1}$ and $P_{2}$ have the same "privileged" exponents, with respect to $<$, and the same $\delta$-exponents, with respect to $<^{\prime}$, that in Example 2 and an analogous argument shows that $\left\{P_{1}, P_{2}\right\}$ isn't a Gröbner base, but it is a Gröbner $\delta$-base of the ideal $I$ for $a=x_{1}, b \in \mathbf{C}\left[\left[x_{1}\right]\right]$ and $d$ a power series in $x_{1}-x_{2}$.

As in Remark 27 we can extend the previous computation to the case where $b \in$ $\mathbf{C}\left[\left[x_{1}, x_{3}, \ldots, x_{n}\right]\right]$ and $d$ is a power series in $x_{1}-x_{2}$ with coefficients in $\mathbf{C}\left[\left[x_{3}, \ldots, x_{n}\right]\right]$.

7. Applications: Flatness and finiteness. As elementary applications of Gröbner $\delta$-bases, we have the effective solution for the ideal membership problem, variable elimination problem and effective intersection of ideals. We can also calculate a generating system of the $\mathcal{D}$-module of syzygies of a finite subset $\left\{P_{1}, \ldots, P_{r}\right\}$ of $\mathcal{D}$, as well as a free resolution of a finitely generated (left) $\mathcal{D}$-module. Calculating free resolutions of a $\mathcal{D}$-module, we have found examples where the use of Gröbner $\delta$-bases is, in some sense, more efficient than the one of Gröbner bases (see Remark 28).

In this section the ring $\mathcal{H}$ is a noetherian sub-k-algebra of

$$
\mathbf{k}((\tilde{X}))=\mathbf{k}\left(\left(x_{1}, \ldots, x_{n}, x_{n+1}, \ldots, x_{n+m}\right)\right),
$$

stable under the action of $\partial_{i}$ for $i=1, \ldots, n$ and satisfying the two additional conditions of Remark 12. We denote as before $\mathcal{D}=\mathcal{H}[\partial]=\mathcal{H}\left[\partial_{1}, \ldots, \partial_{n}\right]$.

The aim of this section is to characterize flatness and finiteness of a $\mathcal{D}$-module by using the notion of Gröbner $\delta$-bases, following the work of A. Assi [ASS-2] in the commutative case.

We can see the quotient $\mathcal{D} / I$ as a family of $A_{n}(\mathbf{k})$-modules, the space of parameters being $\mathbf{C}^{m}$. In this section we will see when this family is flat.

Let $S$ be a multiplicatively closed subset of $\mathcal{H}$. The ring $S^{-1} \mathcal{H}$ is a noetherian sub$\mathbf{k}$-algebra of $\mathbf{k}((\tilde{X}))$, stable under the action of the derivations $\partial_{1}, \ldots, \partial_{n}$ and satisfying the two additional conditions of Remark 12. So, we can consider the sub-k-algebra $S^{-1} \mathcal{D}$ of $\mathbf{k}((\tilde{X}))[\partial]$, generated by $S^{-1} \mathcal{H}$ and $\partial_{1}, \ldots, \partial_{n}$.

One can define in $S^{-1} \mathcal{D}$ the notions of section 2.

Let $I \subset \mathcal{D}$ a left ideal. We denote by $S^{-1} I$ the ideal of $S^{-1} \mathcal{D}$ generated by $I$ and by $i n^{\delta}\left(S^{-1} I\right)$ the ideal (of $\left.S^{-1} \mathcal{H}[\zeta]\right)$ generated by $\left\{i n^{\delta}(P): P \in\left(S^{-1} I\right) \backslash\{0\}\right\}$. Here 
$S^{-1} \mathcal{H}[\zeta]$ denotes the polynomial ring in the variables $\zeta=\left(\zeta_{1}, \ldots, \zeta_{n}\right)$ and coefficients in $S^{-1} \mathcal{H}$. We have:

Proposition 30. Suppose $\left\{P_{1}, \ldots, P_{r}\right\}$ is a Gröbner $\delta$-base of $I$. If $S$ is a multiplicatively closed subset of $\mathcal{H}$ then $i n^{\delta}\left(S^{-1} I\right)$ is generated by $\left\{i n^{\delta}\left(P_{1}\right), \ldots, i n^{\delta}\left(P_{r}\right)\right\}$ in $S^{-1} \mathcal{H}[\zeta]$. In particular, $i n^{\delta}\left(S^{-1} I\right)=S^{-1}\left(i n^{\delta}(I)\right)$ and $\left\{P_{1} / 1, \ldots, P_{r} / 1\right\}$ is a Gröbner $\delta$-base of $S^{-1} I$.

Let $\mathfrak{P}$ be a prime ideal of $\mathcal{H}$. Then $S=\mathcal{H} \backslash \mathfrak{P}$ is a multiplicatively closed subset of $\mathcal{H}$. We denote $\mathcal{H}_{\mathfrak{P}}=S^{-1} \mathcal{H}, \mathcal{D}_{\mathfrak{P}}=S^{-1} \mathcal{D}$ and $I_{\mathfrak{P}}=S^{-1} I$.

For each ideal $\mathcal{K}$ in $\mathcal{H}$, we denote $V(\mathcal{K})=\{\mathfrak{P} \in \operatorname{Spec}(\mathcal{H}): \mathcal{K} \subseteq \mathfrak{P}\}$, which is a Zariski closed subset of $\operatorname{Spec}(\mathcal{H})$. Here we endowed the set $\operatorname{Spec}(\mathcal{H})$ of prime ideals of $\mathcal{H}$ with its Zariski topology.

Let us consider $J=\prod_{i=1}^{s} C(\alpha(i) ; I)$ as an ideal in $\mathcal{H}$, where $\{\alpha(1), \ldots, \alpha(s)\}$ is a $\delta$-stair of the ideal $I$ (see Remark 5). Let us denote $U=\operatorname{Spec}(\mathcal{H}) \backslash V(J)$. We have:

TheOrem 31. With the notations as above, let $\mathfrak{P} \in U$. Then $\mathcal{D}_{\mathfrak{P}} / I_{\mathfrak{P}}$ is a free (and then a flat) $\mathcal{H}_{\mathfrak{P}}$-module.

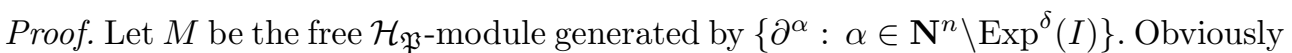
we have $\operatorname{Exp}^{\delta}(I)=\operatorname{Exp}^{\delta}\left(I_{\mathfrak{P}}\right)$. Let us consider a Gröbner $\delta$-base $\left\{P_{1}, \ldots, P_{r}\right\}$ of $I$. By Proposition 30, $\left\{P_{1} / 1, \ldots, P_{r} / 1\right\}$ is a Gröbner $\delta$-base of $I_{\mathfrak{P}}$. Now, applying the reduction algorithm with respect to $\left\{P_{1} / 1, \ldots, P_{r} / 1\right\}$ (see Theorem 15), each $P \in \mathcal{D}_{\mathfrak{P}}$ can be written as a sum

$$
P=P^{\prime}+P^{\prime \prime}
$$

with $P^{\prime} \in I_{\mathfrak{P}}$ and $P^{\prime \prime} \in M$. Here we have used the equality $C\left(\alpha(i) ; I_{\mathfrak{P}}\right)=\mathcal{H}_{\mathfrak{P}}$ for each $i=1, \ldots, s$.

So, we have proved that $\mathcal{D}_{\mathfrak{P}}=I_{\mathfrak{P}}+M$ and it is obvious that $I_{\mathfrak{P}} \cap M=(0)$, so the $\mathcal{H}_{\mathfrak{P}}$-modules $\mathcal{D}_{\mathfrak{P}} / I_{\mathfrak{P}}$ and $M$ are isomorphic. Then $M$ is a free $\mathcal{H}_{\mathfrak{P}}$-module.

Proposition 32. With the notations as above, we have

1. If $C(0 ; I)=I \cap \mathcal{H} \neq(0)$, then $U=\operatorname{Spec}(\mathcal{H}) \backslash V(C(0 ; I))$ is the maximal open set of flatness.

2. If $C(\alpha(k) ; I)=\mathcal{H}$ for each $k \in\{1, \ldots, s\}$, then $\mathcal{D} / I$ is a flat $\mathcal{H}$-module.

Proof. 1. We have $C(0 ; I)=\mathcal{H} \cap I$ (see Remark 11). Suppose $U$ is not maximal, then there exists $\mathfrak{P} \in \operatorname{Spec}(\mathcal{H}) \backslash U$ such that $\mathcal{D}_{\mathfrak{P}} / I_{\mathfrak{P}}$ is $\mathcal{H}_{\mathfrak{P}}$-flat. If $C(0 ; I) \neq(0)$ then $\mathcal{H}_{\mathfrak{P}} \cap I_{\mathfrak{P}} \neq(0)$, which is impossible by flatness of $\mathcal{H}_{\mathfrak{P}}[\partial] / I_{\mathfrak{P}}$ over $\mathcal{H}_{\mathfrak{P}}$.

2, We have $C\left(\alpha_{k} ; I\right)=\mathcal{H}$ for each $\alpha_{k}$ in a $\delta$-stair of $I$. So, we have $U=\operatorname{Spec}(\mathcal{H})$ and then $\mathcal{H}[\partial] / I$ is $\mathcal{H}$-flat.

ExAmple 4. Let us denote $\mathbf{C}[[X]]=\mathbf{C}\left[\left[x_{1}, \ldots, x_{n}\right]\right]$ and consider the ideal of Example 3. Namely, let us consider the differential operators $\left\{P_{1}, P_{2}\right\} \subset \mathcal{D}=\mathbf{C}[[X]]\left[\partial_{1}, \partial_{2}\right]$ where

$$
P_{1}=x_{1} \partial_{1}+x_{1} \partial_{2}+b, \quad P_{2}=\left(x_{2}-x_{1}\right) \partial_{2}-d
$$

with $b \in \mathbf{C}\left[\left[x_{1}, x_{3}, \ldots, x_{n}\right]\right]$ and $d$ a power series in $x_{1}-x_{2}$ with coefficients in $\mathbf{C}\left[\left[x_{3}, \ldots\right.\right.$ $\left.\ldots, x_{n}\right]$ ]. We will also suppose $b$ is a multiple of $x_{1}$. In particular, $\mathcal{D} / I$ is not a flat $\mathbf{C}[[X]]$-module, because the class of $\partial_{1}+\partial_{2}+b / x_{1} \bmod I$ has $x_{1}$-torsion. 
We know by Example 3 that $\left\{P_{1}, P_{2}\right\}$ is a Gröbner $\delta$-base of $I$. Then a $\delta$-stair of $I$ is $\left\{\exp ^{\delta}\left(P_{1}\right), \exp ^{\delta}\left(P_{2}\right)\right\}$, i.e. $\{(1,0),(0,1)\}$.

Moreover, by Theorem 18, we have

$$
C((1,0) ; I)=C\left((1,0) ; P_{1}, P_{2}\right)=\left\langle x_{1}\right\rangle, \quad C((0,1) ; I)=C\left((0,1) ; P_{1}, P_{2}\right)=\left\langle x_{2}-x_{1}\right\rangle .
$$

Let us consider $J=C((1,0) ; I) C((0,1) ; I)$, i.e. $J=\left\langle x_{1}\left(x_{2}-x_{1}\right)\right\rangle$. By Theorem 31 , $\mathbf{C}[[X]]_{\mathfrak{P}}\left[\partial_{1}, \partial_{2}\right] / I_{\mathfrak{P}}$ is a flat $\mathbf{C}[[X]]_{\mathfrak{P}}$-module for $\mathfrak{P} \in U=\operatorname{Spec}(\mathbf{C}[[X]]) \backslash V(J)$.

TheOREM 33. Let $I$ be an ideal of $\mathcal{D}$. The following are equivalent:

1. $\mathcal{D} / I$ is a finitely generated $\mathcal{H}$-module.

2. For each $i=1, \ldots, n$ there exists $a_{i} \in \mathbf{N}$ such that

$$
\alpha(i)=a_{i} \epsilon_{i} \in \operatorname{Exp}^{\delta}(I)
$$

and

$$
C(\alpha(i) ; I)=\mathcal{H},
$$

here $\epsilon_{i}$ is the $i$-th element of the canonical base of $\mathbf{N}^{n}$.

Proof. $1 \Rightarrow 2 .:$ For each $i \in\{1, \ldots, n\}$ we consider the sub- $\mathcal{H}$-module $M \subset \mathcal{D} / I$ generated by the set

$$
\left\{1+I, \partial_{i}+I, \ldots, \partial_{i}^{k}+I, \ldots\right\} .
$$

By the finiteness of $M$ over $\mathcal{H}$, there exists $\alpha(i)=a_{i} \epsilon_{i} \in \operatorname{Exp}^{\delta}(I)$ such that $C(\alpha(i) ; I)=$ $\mathcal{H}$, for some $a_{i} \in \mathbf{N}$.

2. $\Rightarrow 1$.: Let us write $\bar{\Delta}=\mathbf{N}^{n} \backslash \bigcup_{i=1}^{n}\left(\alpha(i)+\mathbf{N}^{n}\right)$. Let us consider $M$ as the $\mathcal{H}$-module generated by the finite set $\left\{\partial^{\alpha}: \alpha \in \bar{\Delta}\right\}$. We have $\mathcal{D}=I+M$ and then $\mathcal{D} / I$ is a quotient of $M$. Thus $\mathcal{D} / I$ is finitely generated as $\mathcal{H}$-module.

\section{References}

[ASS-1] A. Assi, Constructions effectives en algèbre commutative, $\mathrm{PhD}$ thesis, Univ. Grenoble (Joseph Fourier), 1991.

[ASS-2] A. Assi, On flatness of generic projections, J. Symbolic Computation 18 (1994), 447462.

[ACG-1] A. Assi, F. Castro-Jiménez and J.-M. Granger, The Gröbner fan of an $A_{n}$-module, J. Pure Appl. Algebra 150 (2000), 27-39.

[ACG-2] A. Assi, F. Castro-Jiménez and J.-M. Granger, The analytic standard fan of a $\mathcal{D}$ module, J. Pure Appl. Algebra 164 (2001), 3-21.

[BUCH] B. Buchberger, A theoretical basis for the reduction of polynomial to canonical forms, ACM SIGSAM Bull. 39 (1976), 19-29.

[CAS-1] F. J. Castro, Théorème de division pour les opérateurs différentiels et calcul des multiplicités, PhD thesis, Univ. Paris VII, 1984.

[CAS-2] F. J. Castro, Calculs effectifs pour les idéaux d'opérateurs différentiels, in: Travaux en Cours. Géométrie Algébrique et Applications, Tome III, Hermann, Paris, 1987, 1-19.

[CLO] D. Cox, J. Little and D. O'Shea, Ideals, Varieties and Algorithms. An Introduction to Computational Algebraic Geometry and Commutative Algebra, Springer-Verlag, 1992. 
[IN-PA] M. Insa and F. Pauer, Gröbner bases in rings of differential operators, in: Gröbner Bases and Applications, London Math. Soc. L.N.S. 251, Cambridge Univ. Press, Cambridge, 1998, 367-380.

[MOR] M. A. Moreno-Frías, Métodos computacionales en los sistemas de ecuaciones en derivadas parciales, $\mathrm{PhD}$ thesis, Universidad de Sevilla, 2000.

[SAB] C. Sabbah, Proximité évanescente. I. La structure polaire d'un D-module (Appendice en collaboration avec F. Castro), Compositio Math. 62 (1987), 283-328.

[TRI] W. Trinks, Über Buchbergers verfahren, Systeme algebraicher Gleichungen zu lösen, J. Number Theory 10 (1978), 475-488. 